\title{
Freezing thresholds and cirrus cloud formation mechanisms inferred from in situ measurements of relative humidity
}

\author{
W. Haag ${ }^{1}$, B. Kärcher ${ }^{1}$, J. Ström ${ }^{2}$, A. Minikin ${ }^{1}$, U. Lohmann ${ }^{3}$, J. Ovarlez ${ }^{4}$, and A. Stohl ${ }^{5,}{ }^{*}$ \\ ${ }^{1}$ Deutsches Zentrum für Luft- und Raumfahrt (DLR), Institut für Physik der Atmosphäre (IPA), Oberpfaffenhofen, Germany \\ ${ }^{2}$ Stockholm University, Institute of Applied Environmental Research (ITM), Stockholm, Sweden \\ ${ }^{3}$ Dalhousie University, Department of Physics and Atmospheric Science, Halifax, Nova Scotia, Canada \\ ${ }^{4}$ Laboratoire de Météorologie Dynamique (LMD), CNRS-IPSL, École Polytechnique, Palaiseau, France \\ ${ }^{5}$ Lehrstuhl für Bioklimatologie und Immissionsforschung, Technische Universität München (TUM), Freising, Germany \\ *Now at: Cooperative Institute for Research in Environmental Sciences (CIRES), University of Colorado/NOAA Aeronomy \\ Laboratory, Boulder, Colorado, U.S.
}

Received: 24 March 2003 - Published in Atmos. Chem. Phys. Discuss.: 30 June 2003

Revised: 25 September 2003 - Accepted: 25 September 2003 - Published: 27 October 2003

\begin{abstract}
Factors controlling the microphysical link between distributions of relative humidity above ice saturation in the upper troposphere and lowermost stratosphere and cirrus clouds are examined with the help of microphysical trajectory simulations. Our findings are related to results from aircraft measurements and global model studies. We suggest that the relative humidities at which ice crystals form in the atmosphere can be inferred from in situ measurements of water vapor and temperature close to, but outside of, cirrus clouds. The comparison with concomitant measurements performed inside cirrus clouds provides a clue to freezing mechanisms active in cirrus. The analysis of field data taken at northern and southern midlatitudes in fall 2000 reveals distinct differences in cirrus cloud freezing thresholds. Homogeneous freezing is found to be the most likely mechanism by which cirrus form at southern hemisphere midlatitudes. The results provide evidence for the existence of heterogeneous freezing in cirrus in parts of the polluted northern hemisphere, but do not suggest that cirrus clouds in this region form exclusively on heterogeneous ice nuclei, thereby emphasizing the crucial importance of homogeneous freezing. The key features of distributions of upper tropospheric relative humidity simulated by a global climate model are shown to be in general agreement with both, microphysical simulations and field observations, delineating a feasible method to include and validate ice supersaturation in other largescale atmospheric models, in particular chemistry-transport and weather forecast models.
\end{abstract}

Correspondence to: W. Haag

(Werner.Haag@dlr.de)

\section{Introduction}

Understanding the formation of cirrus clouds in the upper troposphere and tropopause region is a necessary prerequisite in developing the ability to understand and predict the radiative effects of cirrus clouds, their role in precipitation processes and in the hydrological cycle, and hence their impact on climate. High-altitude ice clouds are thought to freeze-dry air entering the tropical lower stratosphere, thereby influencing the stratospheric water vapor $\left(\mathrm{H}_{2} \mathrm{O}\right)$ budget (Danielsen, 1982; Jensen et al., 1996); modify heating rates in the tropical tropopause region, thereby influencing stratospheric ozone and the stratospheric circulation (Dessler et al., 1996); lead to vertical redistribution of $\mathrm{H}_{2} \mathrm{O}$ and freezing nuclei, with a potential to modify the development of supercooled low level clouds (Braham, 1967); and trigger heterogeneous chemistry at middle and high latitudes, thereby possibly contributing to the observed decline of ozone in the midlatitude lower stratosphere (Borrmann et al., 1996).

Despite the recognition of the importance of cirrus clouds, details of their formation mechanisms are not very well understood. In particular, the relative humidities over ice, RHI, at which cirrus ice crystals form (hereafter referred to as freezing thresholds) are difficult to infer from in situ measurements, because the freezing of aerosol particles occurs very rapidly (seconds to minutes) and the thermodynamical variables controlling the phase transition are highly variable over the time scale of cloud formation.

The present work uses information about temperature and specific humidity from atmospheric trajectories in conjunction with a microphysical model (Sect. 2 and Appendix) to simulate frequency distributions of RHI with and without prescribed cirrus cloud formation processes (Sect. 3). In particular, the factors controlling the slopes and cut-offs appearing in the distributions of RHI in ice-supersaturated regions 
are examined and related to freezing thresholds and freezing mechanisms, thereby establishing the microphyical link between distributions of RHI and cirrus clouds.

Building on the simulations, this study provides a detailed interpretation of the measurements of RHI outside of and inside cirrus clouds during the Interhemispheric Differences in Cirrus Properties From Anthropogenic Emissions (INCA) field experiment (Ovarlez et al., 2002). Relating the numerical results to field measurements of RHI allows important conclusions to be drawn regarding freezing mechanisms in midlatitude cirrus clouds. By means of global climate model studies we demonstrate that the key features of distributions of RHI in ice-supersaturated regions can be simulated with large-scale models. A discussion relating our findings to long-term aircraft measurements and global satellite observations of RHI is also given. The paper concludes with a short summary of the main results and a brief outlook (Sect. 4).

We expand on previous work (Heymsfield and Miloshevich, 1995; Heymsfield et al., 1998; Pfister et al., 2001; Gettelman et al., 2002) by using more accurate tools to separate measurements of RHI inside and outside of clouds; by presenting new inferences concerning the dependence of the freezing thresholds on properties of freezing atmospheric aerosols and by highlighting differences between freezing mechanisms in cirrus; and by delineating an improved treatment of ice supersaturation and cirrus in global models.

\section{Distributions of relative humidity}

A growing body of in situ observations shows that the upper troposphere and tropopause region at low and middle latitudes are frequently supersaturated with respect to ice (Jensen et al., 2001), allowing the formation and persistence of cirrus clouds and aircraft contrails. The occurrence of thin cirrus clouds extending well into the lower stratosphere in the wintertime polar regions is a well known phenomenon (Kärcher and Solomon, 1999). Recent satellite data provide further compelling evidence for the widespread existence of subvisible cirrus at the tropopause (Spang et al., 2002).

Observations suggest that the distribution of RHI in ice supersaturated regions follows an almost exponential decay, indicating that the underlying processes that cause this functional dependence act in a statistically independent manner (Gierens et al., 1999). The question arises of how realistic probability distributions of RHI can be generated with the help of a model to be studied systematically.

We suggest that such distributions are primarily brought about by vertical air motions that cause a certain distribution of atmospheric temperatures $T$. Variability of $\mathrm{H}_{2} \mathrm{O}$ also plays a role, but is less influential. When distributions of $T$ and $\mathrm{H}_{2} \mathrm{O}$ concentrations are combined to derive RHI, the resulting distributions of RHI exhibit a quasi-exponential decrease towards high RHI values, in agreement with observations. A more detailed investigation of these basic aspects is presented in a separate study (Kärcher and Haag, 2003).

In the present work, we compute fields of RHI by advecting $\mathrm{H}_{2} \mathrm{O}$ along domain-filling atmospheric trajectories and allowing ice formation and evaporation, that is, the formation and disappearence of cirrus clouds. We use an aerosol-cirrus model (the Advanced Particle Simulation Code, APSC) along each trajectory that includes detailed particle microphysics and an approximate treatment of the loss of ice crystals through sedimentation. We focus on cold cirrus that form directly from freezing aerosol particles. Different aerosol freezing thresholds are imposed in the model to investigate their impact on the distributions of RHI.

In air that is subsaturated with respect to the ice phase (more precisely, up to RHI=95\%), we use $T$, the air pressure, and the specific humidity directly from the European Centre for Medium-Range Weather Forecasts (ECMWF) model to calculate the time evolution of RHI along the trajectories with the APSC. The ECMWF has been shown to provide RHI fields below ice saturation in good agreement with in situ data (Ovarlez and Velthoven, 1997).

However, the ECMWF model, as most other global models, does not predict ice supersaturation (Lohmann and Kärcher, 2002). For this reason, at RHI values above $95 \%$, we keep the $\mathrm{H}_{2} \mathrm{O}$ mixing ratio fixed on each trajectory and let RHI vary according to changes of $T$, air pressure, and the calculated rates of $\mathrm{H}_{2} \mathrm{O}$ deposition onto and evaporation from aerosol and cirrus ice particles. The $\mathrm{H}_{2} \mathrm{O}$ mixing ratio is reinitialized from the actual ECMWF fields after the cirrus cloud vanishes (typically forced by synoptic warming). If cirrus formation does not occur within 6 hours after RHI first increased above $95 \%$ (because the prescribed freezing thresholds are not surpassed), the $\mathrm{H}_{2} \mathrm{O}$ mixing ratio is likewise reinitialized. This procedure allows us to predict realistic RHI fields in cirrus-forming regions.

The APSC calculations are based on a subset of trajectories within the tropopause region with mostly cold $(<235 \mathrm{~K})$ temperatures (see Appendix). From this subset, we have removed all RHI data belonging to temperatures outside the range of temperatures measured during INCA, to enable a proper comparison between calculated and observed $f(\mathrm{RHI})$. The mean temperature of the remaining data in the computational domain is $223 \mathrm{~K}$. This is close to the observed mean value near $225 \mathrm{~K}$. We have also checked that the minimum temperatures $(\sim 211-216 \mathrm{~K})$ were low enough and the observed temperature distributions had sufficient variability to produce RHI values above the homogeneous freezing thresholds in both campaigns (Kärcher and Haag, 2003).

The trajectories follow the motions caused by atmospheric waves with wavelengths sufficiently long to be resolved by the ECMWF model. However, smaller-scale gravity waves, non-hydrostatic waves, and deep convection are not explicitly resolved. The trajectories reflect these processes only to the extent that the physical parameterizations feed back on the grid-scale winds, generally underestimating mesoscale 
temperature variability along the trajectories. To account for mesoscale dynamic features, we superimpose rapid temperature oscillations to the ECMWF-based, synoptic temperatures, with random amplitudes (up to $2 \mathrm{~K}$ ) and mean periods ( $\sim 20 \mathrm{~min}$ ) consistent with the INCA measurements (Kärcher and Ström, 2003).

More details of the employed meteorological fields, the trajectory model, and the microphysical simulations including some technical issues are outlined in the Appendix.

\section{Results and discussion}

We work out the main features of the RHI distribution and study their microphysical link to cirrus clouds (Sects. 3.1 and 3.2). To provide a link to observations and global simulations, we relate our findings to the INCA field experiment (Sect. 3.3), to simulations of RHI carried out with the general circulation model (GCM) ECHAM (Sect. 3.4), and to longer-term aircraft- and satellite-based measurements of RHI (Sect. 3.5).

\subsection{Distributions of RHI outside of cirrus}

Figure 1 displays the calculated distributions of relative humidity over ice, $f(\mathrm{RHI})$, outside but near cirrus clouds (left panel) and inside cirrus clouds (right panel). We first consider a hypothetical case (labelled ADV in the left panel) where ice formation was not allowed in the APSC, representing pure advection of $\mathrm{H}_{2} \mathrm{O}$ mixing ratios.

The distribution ADV (purple curve) shows a continuous, quasi-exponential decrease of RHI somewhat above saturation. It has been shown analytically that the slope of $f(\mathrm{RHI})$ in case ADV is mainly determined by the spread of the distribution of temperatures and by the properties of the saturation vapor pressure curve over ice, or equivalently, the ClausiusClapeyron equation (Kärcher and Haag, 2003). The probability to find a given supersaturation with respect to ice is primarily determined by the subset of upward moving trajectories on which cooling of air parcels carrying relatively high $\mathrm{H}_{2} \mathrm{O}$ content from lower tropospheric levels generate high RHI values. As the underlying cold ECMWF tropopause temperatures exhibit a large variability, the resulting distribution is rather flat.

To study the impact of cirrus formation on the cloudcleared distributions, we allowed the nucleation of ice in the simulations and collected all data points where no cirrus particles were present. As described in the Appendix, we vary the ice nucleation properties of the freezing aerosol. In the first type of calculations (HOM, red curves in Fig. 1), pure homogeneous freezing of supercooled $\mathrm{H}_{2} \mathrm{SO}_{4} / \mathrm{H}_{2} \mathrm{O}$ aerosols is considered. The assumption of aqueous $\mathrm{H}_{2} \mathrm{SO}_{4}$ as freezing aerosols is not crucial, as the experimental uncertainty in RHI would not allow to resolve activity-induced (non-equilibrium) differences in inferred freezing thresh- olds. In the second type (MIX $n$, black and green curves), competition of homogeneous nuclei with few $(n=0.1$ and $0.001 \mathrm{~cm}^{-3}$ ) but more efficient ice nuclei (IN) during ice initiation is allowed. In the third type (HET, blue curves), we consider pure heterogeneous freezing of all aerosol particles.

The prescribed nominal IN freezing threshold (defined in the Appendix) was $130 \%$ in cases MIX and HET. In principle, the heterogeneous freezing threshold could lie anywhere between $100 \%$ and the homogeneous freezing limit; this specific choice is motivated by the field experiments discussed later in Sect. 3.3. Apart from this, it is not necessary to specify the chemical nature of the IN in the model.

The most obvious change of the cloud-free $f(\mathrm{RHI})$ (left panel in Fig. 1) introduced by ice formation compared to case ADV is the appearance of a cut-off in the supersaturated region. In case HOM (MIX/HET), no data points above $\sim 147.5 \%(\sim 127.5 \%)$ are found outside of clouds. The cutoff is the value of RHI where the very first ice crystals form from freezing aerosol particles. The calculated cut-offs are averages over all individual ice formation events, involving different cooling rates and therefore different portions of the freezing particle size distribution. They are not perfectly sharp, because of aerosol size effects and the action of waves that change RHI during freezing, especially in the cases MIX and HET, and the dependence of the nominal freezing thresholds on $T$ (Koop et al., 2000a) in case HOM. As we have only considered model output every $360 \mathrm{~s}$ (see Appendix), the exact homogeneous cut-offs are slightly (up to $\sim 2 \%$ ) larger than those reported here, as a finer output would better capture the absolute maximum RHI prior to nucleation.

There is no significant difference in the distributions MIX and HET outside of clouds, because in both cases the same freezing properties have been assumed for the IN from which the first ice particles form. If we prescribed lower or higher IN freezing thresholds, the cut-off in the cases MIX and HET would shift accordingly to lower or higher values. The total number density $n$ of IN present does not affect the onset of freezing in the cases MIX $n$. By implication, the locations of the cut-offs do not depend on the choice of the trajectory space and time domain or on details of the cirrus cloud microphysics other than the lowest freezing thresholds.

We have argued earlier in Sect. 3.1 that in case ADV the slope of $f(\mathrm{RHI})$ is mainly determined by the ClausiusClapeyron equation. How does the change of the slopes between the different cases shown in the left panel of Fig. 1 comes about?

As we sample out-of-cloud points to determine $f(\mathrm{RHI})$, the cirrus clouds themselves cannot alter the properties of the distributions between ice saturation and the cut-off. However, cirrus formation and persistence (and implicitly, ice crystal depositional growth and sedimentation) indirectly affects the slope of $f(\mathrm{RHI})$. In case HOM, all data points above $147.5 \%$ are turned from out-of-cloud into in-cloud data points. They are considered in case ADV, but no longer in the HOM data set. As the neglected data points belong to 

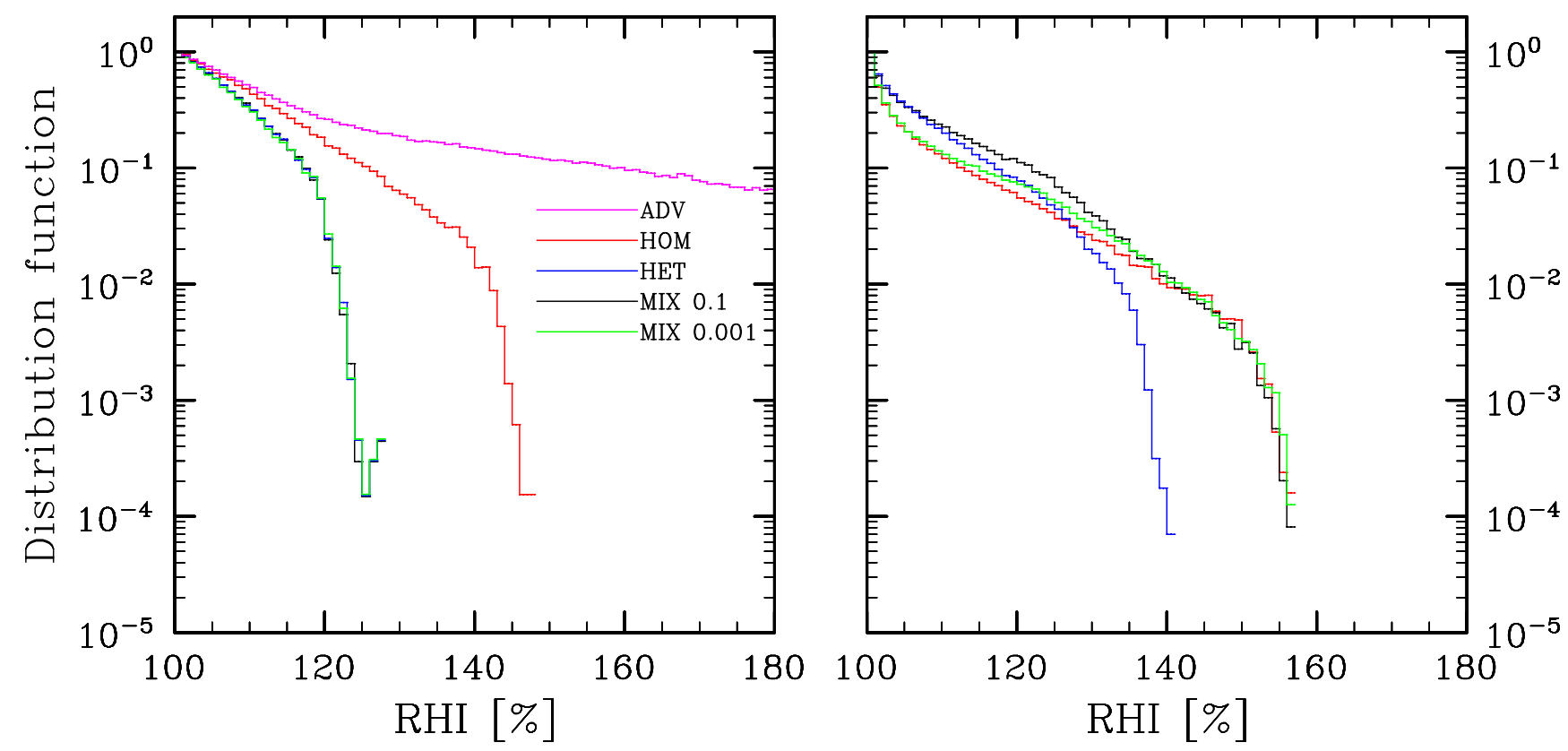

Fig. 1. Calculated distributions of RHI above ice saturation outside of (left panel) and inside (right panel) cirrus clouds. The distributions are normalized with the number of data points in the respective $100 \%$ bin and all RHI values were binned into $1 \%$ intervals. Liquid aerosol particles and heterogeneous ice nuclei freeze with different nominal ice nucleation thresholds (MIX $n, n$ denoting the total concentrations of ice nuclei in $\mathrm{cm}^{-3}$ ); the entire particle distribution freezes homogeneously (HOM), heterogeneously (HET), or not at all (ADV, left panel only). For details see text.

trajectories that primarily produce large supersaturations and eventually lead to ice nucleation, the slope increases when homogeneous freezing is considered. The same selection effect further increases the slope in the cases MIX and HET, as even more such data points along cloud-forming trajectories (all above 127.5\%) are excluded from the analysis.

Summarizing the findings concerning distributions of RHI in ice-supersaturated regions close to, but outside of, cirrus clouds, we have shown that (1) the cut-off is directly related to the onset of freezing in cirrus clouds (the relative humidity where the first ice crystals form) and (2) the slope is controlled by a combination of temperature variations and cirrus microphysical processes (including ice crystal growth and sedimentation). Because of point (2), a quantitative comparison of the slopes of modeled and measured RHI distributions is difficult, unless the meteorological wind fields and physical relations governing cirrus formation, growth, and persistence are well captured by models. We reiterate this issue in Sects. 3.3 and 3.4 .

\subsection{Distributions of RHI inside cirrus}

The modeled distributions of RHI inside cirrus are shown in the right panel of Fig. 1. This data set is complementary to that shown in the left panel, as now all data points where cloud particles were present have been collected from the simulations.
All distributions fall off rapidly above saturation and exhibit rather sharply marked peak values. The cut-off of the in-cloud distribution is the maximum value of RHI reached during cloud formation. All ice crystals nucleate between the out-of-cloud and in-cloud cut-offs (Kärcher and Lohmann, 2002, their Fig. 1). In case HOM/MIX (HET), no data points above $\sim 156.5 \%(\sim 140.5 \%)$ are found inside the clouds.

The difference between the out-of-cloud and in-cloud cutoffs in the absence of wave activity for an aerosol that freezes solely homogeneously is of the order of several percent depending on cooling rates and temperatures (Haag et al., 2003). The same holds for an aerosol that freezes solely heterogeneously. The larger differences in the range 9\% (13\%) seen in the present calculation HOM (HET) are caused by the high cooling rates introduced by the mesoscale temperature oscillations. Such oscillations are capable of generating transient, high peak values of RHI, even in the presence of ice crystals in the cloud formation stage (Kärcher, 2003).

An exception seem to be the MIX cases, where the difference between the out-of-cloud and in-cloud cut-offs are even larger (29\%). This holds for the two IN concentrations considered here in cases MIX 0.1 and MIX 0.001. This is easily explained by the fact that the few IN that freeze first, and thus determine the low out-of-cloud cut-off value, cannot prevent homogeneous freezing of the more abundant liquid aerosols from occurring at the prevailing cooling rates. Subsequent freezing of the liquid particles determines the high in-cloud 
cut-off value. We will show in Sect. 3.3 that the MIX cases provide a clue to the interpretation of the INCA field measurements.

The slope of the in-cloud distributions is controlled by the microphysical processes affecting the interactions of $\mathrm{H}_{2} \mathrm{O}$ molecules with cloud particle surfaces and by cloud dynamical factors such as dilution or turbulence not resolved in our model. When sampling RHI data points in a young cirrus cloud, where ice particles are still growing at high supersaturations, the time needed to complete the growth and approach saturation is longer the lower the temperature. Hence the probability to sample supersaturated data points increases. We thus may expect generally flatter distributions at colder temperatures and increasing average RHI values inside cloud.

In this context we like to stress that in actual atmospheric measurements, the shape of the in-cloud RHI distribution is also affected by the sampling strategy. It is not a universal function as is the cloud-free distribution which follows a quasi-exponential decay (Kärcher and Haag, 2003). Measurements that primarily probe formation regions and air parcels containing freshly nucleated cloud particles will show more supersaturated events than measurements carried out mostly in thick, developed cloud elements.

Summarizing the findings concerning distributions of RHI in ice-supersaturated regions inside cirrus clouds, we have shown that (1) the cut-off of the in-cloud distribution represents the peak value of RHI reached during cloud formation and (2) the difference between out-of-cloud and in-cloud cutoffs is directly related to the aerosol-induced freezing mechanism in cirrus (pure freezing or competition between particles with different freezing properties).

\subsection{Interpretation of the INCA measurements}

\subsubsection{Overview and data analysis}

Two field campaigns were performed during INCA, one in the midlatitude southern hemisphere (SH) out of Punta Arenas, Chile, and one in the midlatitude northern hemisphere (NH) out of Prestwick, Scotland, under comparable meteorological conditions in local autumn in the year 2000. Throughout this work, we use the abbreviations $\mathrm{NH}$ and $\mathrm{SH}$ only to distinguish between the two locales. We do not claim that the two data sets represent conditions covering other parts of the hemispheres or other seasons.

Water vapor mixing ratios were measured in situ by a frostpoint hygrometer and these measurements were converted into RHI using the measured air temperature and pressure. Details of these measurements are reported elsewhere (Ovarlez et al., 2002; Kärcher and Haag, 2003). We excluded all data points above water saturation and above $235 \mathrm{~K}$ to focus on cold cirrus that form by freezing of aerosol particles, as in the model analysis, recall Sect. 3.1. This also removes a few events (spikes) where the RHI taken inside cloud surpassed water saturation at almost constant temperatures: these high values were likely caused by sampling and evaporation of cloud particles in the instrument.

The absolute uncertainty, or precision, of RHI is $\pm 3 \%$ (one standard deviation, $1 \sigma$ ), so that the scatter in the RHI observations is primarily caused by natural variability. Because the conditions during the two campaigns were comparable, a possible bias of the RHI measurements would shift all deduced locations of cut-offs (and hence, inferred freezing tresholds) by the same amount, but does not affect the conclusions about differences in the freezing mechanisms. An indication that the bias in RHI data is small is given in Sect. 3.3.2. The increase in RHI due to evaporation of small (diameter $1 \mu \mathrm{m}$ ) ice crystals or water-rich aerosol particles in the air inlet is estimated to be well below $1 \%$.

The aircraft flight patterns were designed to probe young cirrus clouds (Kärcher and Ström, 2003, their Sect. 2). Our criterion to distinguish between data points inside and outside of cirrus clouds was more conservative than the one based on cloud extinction used by Ovarlez et al. (2002). Here we define a data point as being outside of clouds when the ice particle number density $n_{i}$ measured by the Counterflow Virtual Impactor (CVI) was below $n_{c}=0.009 \mathrm{~cm}^{-3}(\mathrm{NH})$ and below $n_{c}=8 \times 10^{-4} \mathrm{~cm}^{-3}(\mathrm{SH})$. An explanation for the particular choices of $n_{c}$ is given later in Sect. 3.3.3.

The data are archived at $1 \mathrm{~Hz}$ time resolution in the INCA data base. The hygrometer has a response time of $\sim 4 \mathrm{~s}$. We have checked that the analyzed distributions do not significantly change when we applied the criterion $n_{i}<n_{c}$ for 4 consecutive seconds. Further, the $f(\mathrm{RHI})$ remain similar when 4 s-means of RHI are calculated, or when 4 s-means of $T$, air pressure, and $\mathrm{H}_{2} \mathrm{O}$ mixing ratio are calculated and $\mathrm{RHI}$ is derived from these values. We have binned the data into $4 \%$ intervals to roughly account for the experimental uncertainty. The use of other bin widths (up to $8 \%$ ) just leads to more or less data smoothing, leaving average slopes and locations of the cut-offs almost unchanged.

\subsubsection{Freezing thresholds and freezing mechanisms}

The resulting distributions derived from the INCA data as outlined in Sect. 3.3.1 are shown in Fig. 2. As in the model results displayed in Fig. 1, the distributions outside of and inside clouds are shown in the left and right panel, respectively.

A comparison with the modeled out-of-cloud $f$ (RHI) (left panel in Fig. 1) reveals a good agreement between the location of the cut-offs, or freezing thresholds, within the measurements uncertainties ( $3 \sigma$-limits). The model cut-offs appear at $127.5 \%$ (MIX/HET) and at $147.5 \%$ (HOM), marked with arrows in Fig. 2. In the observations, they are centered at $132 \%(\mathrm{NH}$, black curve) and $156 \%$ (SH, red curve).

The nominal homogeneous freezing thresholds at the mean INCA temperature $(225 \mathrm{~K})$ for freezing of particles with radii between 0.05 and $5 \mu \mathrm{m}$ within 1 min range between $146 \%$ and $150 \%$, based on laboratory measurements 

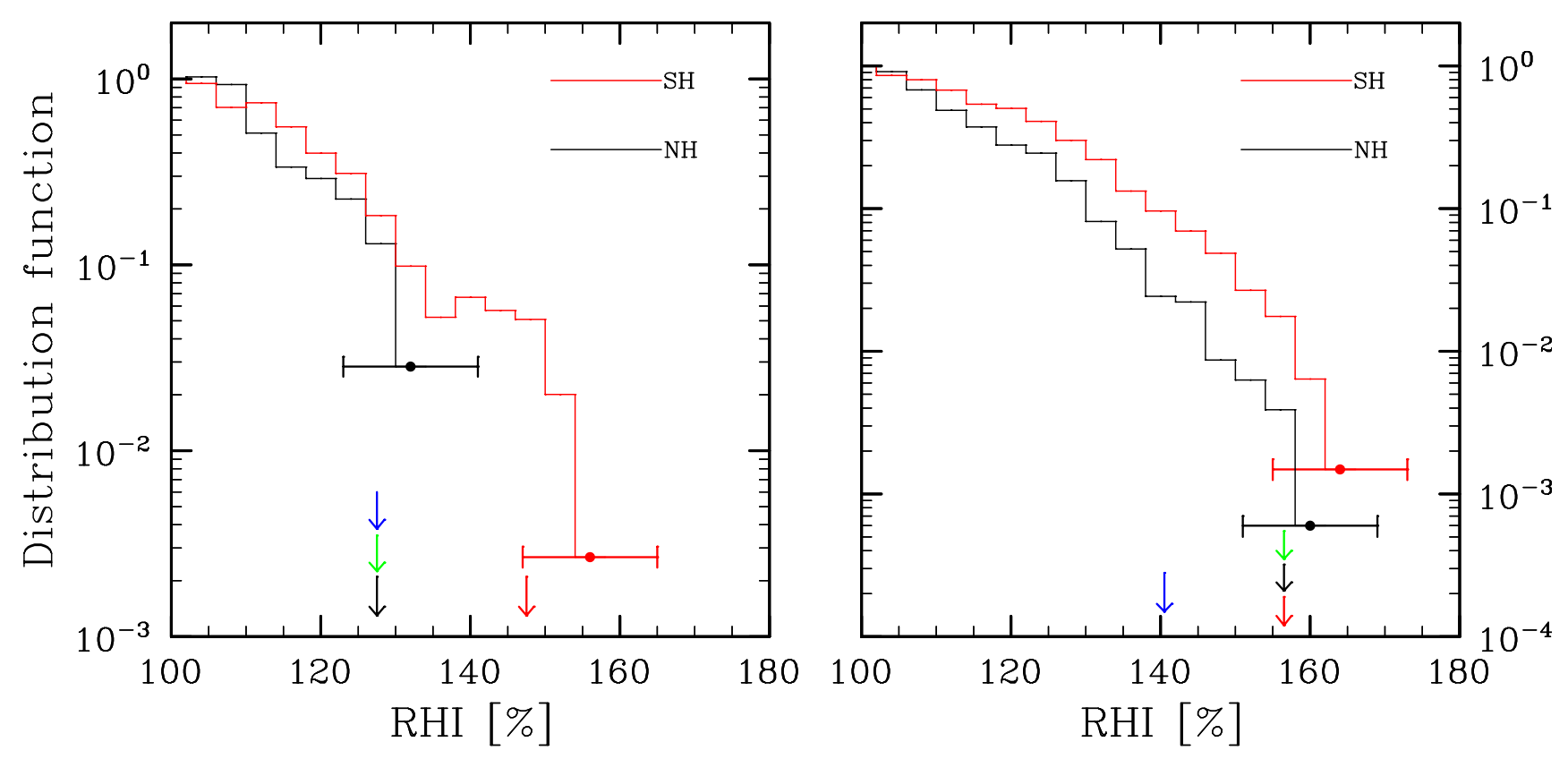

Fig. 2. Distributions of RHI above ice saturation outside of (left panel) and inside (right panel) cirrus clouds measured during INCA in Punta Arenas (SH) and in Prestwick (NH). The distributions are normalized with the number of data points in the respective $100 \%$ bin and all RHI values were binned into $4 \%$ intervals. The precision of the RHI measurements is $\pm 3 \%$ ( $1 \sigma$-limits), with horizontal bars depicting the $3 \sigma$-limits. The colored arrows mark the cut-offs derived from the modeled distributions, taken from Fig. 1 (HOM: red, HET: blue, MIX 0.1: black, MIX 0.001: green).

assuming thermodynamic equilibrium between the freezing droplets and the $\mathrm{H}_{2} \mathrm{O}$ vapor (Koop et al., 2000a). It is known that non-equilibrium effects in rapidly cooling air parcels can increase these freezing thresholds by several percent (Haag et al., 2003), this increase becoming larger with decreasing temperature.

In the model case HOM, we assume homogeneous freezing as the underlying cirrus formation mechanism. This implies a satisfactory agreement between the freezing thresholds deduced from the INCA SH data and the APSC HOM results, and establishes high confidence in the RHI measurements. In particular, the RHI data might have only a small bias towards lower values, likely within about $5 \%$.

The heterogeneous (HET) or mixed (MIX) freezing mechanisms nicely explain the observed, significantly lower cutoff in the NH data set. Recall that contrary to case HOM, the nominal freezing thresholds in the APSC cases MIX and HET have been chosen on purpose to come into good agreement with the $\mathrm{NH}$ observations. Importantly, the $\mathrm{NH}$ data cannot be explained by pure homogeneous freezing of solution droplets, as cut-off values outside the $3 \sigma$-limits $132 \pm 9 \%$ $(\mathrm{NH})$ and $156 \pm 9 \%(\mathrm{SH})$ are extremely unlikely. As we will show later in this section, the case HET cannot explain the observations taken inside clouds.

While we cannot compare the slopes of the calculated and observed distributions quantitatively as explained at the end of Sect. 3.1, we find another remarkable agreement between model and measurements with regard to the relative differ- ence in the slopes. In both cases, the slope of the NH data (cases MIX or HET) is steeper than the slope of the SH data (case HOM). This strongly increases confidence in our proposed interpretation of RHI cut-offs taken outside of cirrus clouds with respect to freezing thresholds.

We now focus attention on $f$ (RHI) taken inside clouds (right panels in Figs. 1 and 2). In the SH (NH) data set, the cut-off is centered at $164 \%(160 \%)$. In the SH measurements, the mean cut-off found inside clouds is $8 \%$ above the cut-off found outside of clouds. This is consistent with the APSC results in case HOM, where a difference of $9 \%$ is predicted.

Hence we may conclude that homogeneous freezing was the predominant pathway to form cirrus during the SH campaign. However, this does not necessarily imply that all freezing particles were supercooled liquid droplets. Part of the freezing aerosol in the SH may have consisted of heterogeneous IN which had freezing thresholds smaller but close to the homogeneous limits (see also Sect. 3.3.3). Such poor IN could possibly alter the core particles that remain from evaporating ice crystals, but would not significantly affect the observed cut-offs.

The situation is completely different in the NH measurements. The observed mean cut-off $\sim 160 \%$ inside clouds is significantly above the mean cut-off $\sim 132 \%$ found outside of clouds. This marked difference of $28 \%$ cannot be explained by experimental uncertainty, and is not consistent with the APSC results from case HET, in which the difference is only $13 \%$. The model assumption in case HET regarding the 
Table 1. Observed (INCA) and calculated (APSC) cut-off relative humidities over ice of the distributions of RHI oustide of and inside cirrus clouds. The SH and NH observations compare well with the model cases HOM and MIX, respectively. No distinction is made between the cases MIX 0.1 and MIX 0.001 because the cut-offs are identical (see Fig. 1).

\begin{tabular}{cccccc}
\hline & \multicolumn{2}{c}{ INCA } & \multicolumn{3}{c}{ APSC } \\
RHI, \% & SH & NH & HOM & MIX & HET \\
\hline outside & 156 & 132 & 147.5 & 127.5 & 127.5 \\
inside & 164 & 160 & 156.5 & 156.5 & 140.5 \\
difference & 8 & 28 & 9 & 29 & 13 \\
\hline
\end{tabular}

heterogeneous freezing mechanism (pure heterogeneous nucleation of ice in chemically uniform aerosols) is incorrect.

However, the NH observations can be fully explained by the two model cases MIX 0.1 and MIX 0.001, irrespective of the assumed IN concentrations. Here, the calculated cut-off inside clouds is significantly $(29 \%)$ above the calculated cutoff outside of clouds, as observed in the NH measurements.

We have summarized observed $(\mathrm{SH}, \mathrm{NH})$ and modeled (HOM, MIX, HET) cut-off relative humidities in Table 1, for convenience. The agreement between the cases HOM and $\mathrm{SH}$ on the one hand and the cases MIX and NH on the other hand in terms of differences between cut-offs inside and outside of cloud is very remarkable. In the APSC case MIX, we could have optimized the agreement outside cloud by chosing a slightly higher (by $\sim 4 \%$ ) nominal freezing threshold. In the other cases where homogeneous freezing is relevant, we have no such freedom in the model as the nominal homogeneous freezing thresholds are determined from laboratory data (Koop et al., 2000a).

It appears that the cut-offs predicted by the APSC in case HOM are close to the lowest possible values consistent with the SH measurements, see red arrows in Fig. 2. We identify five reasons, perhaps partly compensating each other, that could improve the agreement between model and observations. (1) The coarse model output of $3 \mathrm{~min}$, which is of the order of the duration of many freezing events, misses the peak values for the calculated cut-offs. (2) The calculated cut-offs increase when using the same $n_{c}$ in the APSC to perform the cloud clearing as in the observations, instead of $n_{c}=0$ which is a much more stringent criterion. (3) The simulation likely misses some of the variability in the dynamics and aerosol properties present in the observations. Points (1)-(3) would lead to an increase of the modeled cut-offs. Point (2) is further elucidated in Sect. 3.3.3. (4) The measurements of RHI could have a positive bias. (5) The CVI does not detect ice crystals when they are smaller than $5 \mu \mathrm{m}$ in diameter (see Sect. 3.3.3). Points (4) and (5) would cause the real cut-offs to be lower than those deduced from the RHI data. Point (5) is used by Ström et al. (2003) to infer the freezing thresholds from cloud occurrence data taken with the Polar Nephelometer probe.

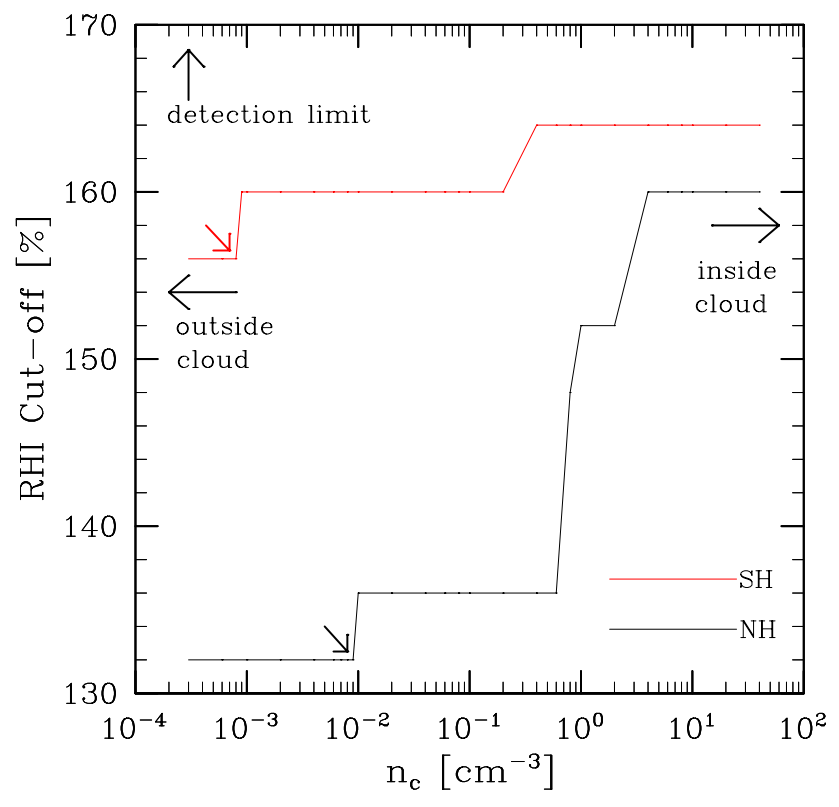

Fig. 3. Cut-off values of the observed distributions of RHI as a function of the cloud-clearing parameter $n_{c}$. The value of $n_{c}$ are based on CVI measurements of the (total) ice crystal concentration, with a detection limit near $3 \times 10^{-4} \mathrm{~cm}^{-3}$. The black and red curves show the transition in cut-offs between out-of-cloud values for $n_{c}<8 \times 10^{-4} \mathrm{~cm}^{-3}$ (SH, red diagonal arrow) and $n_{c}<0.009 \mathrm{~cm}^{-3}$ (NH, black diagonal arrow) to in-cloud values for $n_{c}>0.4 \mathrm{~cm}^{-3}$ (SH) and $n_{c}>4 \mathrm{~cm}^{-3}(\mathrm{NH})$, respectively. As in Fig. 2, all RHI values are binned into $4 \%$ intervals, roughly accounting for data accuracy. The step-like curves become smoother when finer bins are chosen.

In summary, cirrus formation in the $\mathrm{SH}$ is consistent with homogeneous freezing of particles exhibiting a freezing behavior very similar to that of supercooled liquid aerosols. Freezing in the NH was likely initiated by a limited number of efficient IN that triggered ice formation well below the homogeneous limit, explaining the comparatively low cutoffs measured outside of clouds. Our simulation results of the cases MIX demonstrate that the rapid cooling rates prevailing during the campaign were high enough on average to frequently activate homogeneous nucleation in the much more abundant supercooled aerosol particles, in addition to the few IN. This explains why the cut-offs measured inside clouds were near the homogeneous limit in most cases.

\subsubsection{Further analysis and interpretation}

\section{Investigating the transition from cloud-free to cloudy cases}

In Fig. 3 we show cut-off relative humidities over ice as a function of the ice crystal number concentration $n_{c}$ above which we define a measurement to be an in-cloud measurement. This definition depends on the instrument used to probe the clouds, as discussed in more detail by Ström et al. (2003). 


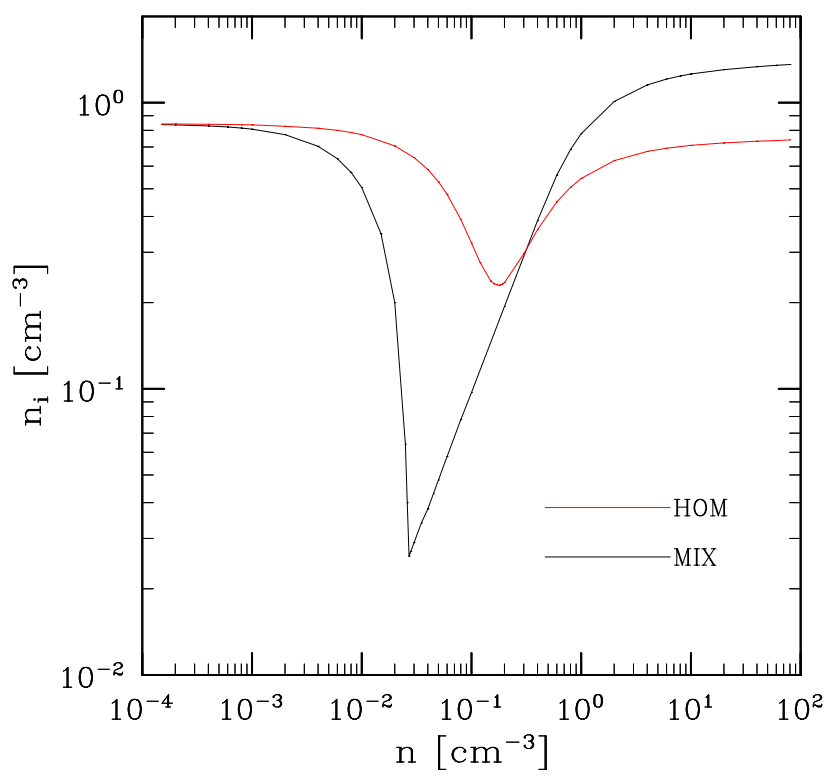

Fig. 4. Total number density of ice crystals $n_{i}$ as a function of the concentration $n$ of large mode aerosol particles calculated by the APSC at average INCA conditions using a bimodal freezing aerosol. The only difference between the cases HOM (red curve) and MIX (black curve) is the assumed freezing threshold of the large mode particles, which is near 152\% (HOM) and 130\% (MIX). The minimum $n$ in case MIX denotes the upper limit IN concentration below which homogeneous freezing still takes place, albeit with reduced $n_{i}$. The reduction of $n_{i}$ due to the presence of IN becomes small for $n<0.01 \mathrm{~cm}^{-3}$, consistent with the NH observations. At the minimum, homogeneous freezing is completely shut off in case MIX.

The values $n_{c}$ used to produce Fig. 3 are based on the CVI measurements. The CVI detects particles with aerodynamic diameters between 5 and $60 \mu \mathrm{m}$; most ice crystals fall in this size range, as we have checked by inspecting the campaignaveraged size distributions, so the CVI data approximate the total number of ice crystals in young cirrus clouds. We point out that the employed CVI is especially sensitive to rather low concentrations of small ice crystals. This feature, along with the sampling strategy to focus on young cirrus, is required to effectively clear the INCA data from cloud events.

The cut-offs shown in Fig. 3 do not change from to the CVI detection limit (see arrow in the upper left corner) up to the $n_{c}$ values indicated by the diagonal arrows on each curve (SH: $n_{c}=8 \times 10^{-4} \mathrm{~cm}^{-3}$ and NH: $n_{c}=0.009 \mathrm{~cm}^{-3}$ ). We have used these $n_{c}$ values as criteria to produce the distributions of RHI shown in Fig. 2 to capture cut-offs as close to the true freezing thresholds as possible and at the same time to maximize the number of data available to produce the distributions. Using lower $n_{c}$ values would reduce the number of RHI points and thus increase the statistical error, but would not affect the cut-offs.

The cut-offs in both data sets increase when the $n_{c}$ criterion is relaxed, approaching pure inside cloud values on the right hand side of the figure. At this point, $n_{c}$ is of the same order as the average number of ice crystals measured during INCA (Kärcher and Ström, 2003). Figure 3 underlines the importance of using an accurate tool to separate measurements outside from inside cloud.

The analysis of $f(\mathrm{RHI})$ presented in Sect. 3.3.2 is based on the premise that the CVI typically probed cloud-forming regions, i.e., young cirrus clouds. When ice crystals have grown to many tens of $\mu \mathrm{m}$ size, they may sediment into lower layers of air. These layers could be (slightly) supersaturated. In such cases, we would measure RHI above $100 \%$ along with some low value of $n_{i}$ (because only few crystals grow large and fall), and this RHI could be misinterpretated as a freezing threshold. (Note that this misinterpretation is not possible in our model owing to the simplified treatment of sedimentation, but must be avoided in more detailed cloudresolving simulations.)

While we cannot fully exclude the possibility of having probed sedimenting ice crystals without the simultaneous presence of high number densities of small crystals, we believe that these cases were rare and that sedimentation affected only a small fraction of the data set (Kärcher and Ström, 2003, their Sect. 3.4, point 2.). Also, if sedimentation was important, we would expect to find more variability in the deduced cut-offs, but the data show a rather stable freezing threshold of $130 \%$ (see also Fig. 5).

\section{Constraining IN concentrations in the NH measurements}

We try to constrain the prescribed number density $n$ of IN in case MIX $n$. It has been shown earlier that heterogeneous IN could suppress homogeneous nucleation under two assumptions (Kärcher and Lohmann, 2003). (1) Ice nuclei must be present in addition to liquid aerosol particles, and both types of particles are, at least to some degree, externally mixed. (2) The peak number of IN present must exceed a critical value, this value depending on the IN freezing threshold, on temperature, and on the vertical wind speed.

Based on these facts, we have performed APSC simulations of cirrus formation in adiabatically rising air parcels, at the average INCA temperature $(225 \mathrm{~K}$, the initial value used in the simulations that start at ice saturation) and updraft speed $\left(25 \mathrm{~cm} \mathrm{~s}^{-1}\right)$ and using the same bimodal aerosol parameters as described in the Appendix. The only variable parameter is the prescribed concentration $n$ of IN. While in all other APSC results discussed in this work the large mode concentration $n$ has been fixed to $0.1 \mathrm{~cm}^{-3}$ in case HOM, here we vary $n$ also in case HOM to enable a proper comparison between HOM and MIX $n$. In Fig. 4 we plot the results for the cases HOM (red curve) and MIX $n$ (black curve), with $n$ varied in the range $10^{-4}-100 \mathrm{~cm}^{-3}$. This range is very large in order to properly explain the physics underlying these simulations. The cases MIX $n$ again assume a nominal IN freezing threshold of $130 \%$ for particles residing in the large mode, while all particles freeze homogeneously in case 
HOM regardless of size, so this remains the only difference between the simulations shown in Fig. 4.

We first discuss the HOM case (red curve). For low number densities of particles in the large mode $\left(n<10^{-3} \mathrm{~cm}^{-3}\right)$, $n$ is too small to affect the freezing process. The freezing aerosol size distribution is practically unimodal; only particles in the small mode centered at $18 \mathrm{~nm}$ radius freeze, producing $n_{i} \simeq 0.83 \mathrm{~cm}^{-3}$ ice particles. When $n$ increases, the mean radius of the portion of the aerosol size distribution that freezes increases. The freezing aerosol size distribution becomes bimodal. At the prescribed conditions (temperature, cooling rate, size distribution), this causes $n_{i}$ to decrease (Kärcher and Lohmann, 2002, their Sect. 2.4.4), until a minimum develops at $n \simeq 0.2 \mathrm{~cm}^{-3}$. Around the minimum, $n_{i} \approx n$; increasing $n$ further leads to a proportional increase of $n_{i}$ with $n$, because now only particles from the large mode centered at $420 \mathrm{~nm}$ radius freeze. The mean radius of the freezing aerosols does not change any more, and the freezing aerosol size distribution becomes unimodal again. The only change now is the increase in the number of large mode particles, which increases $n_{i}$ (Kärcher and Lohmann, 2002, their Sect. 2.4.5). For $n>10 \mathrm{~cm}^{-3}, n_{i}$ saturates to a value of $0.73 \mathrm{~cm}^{-3}$. The change in $n_{i}$ due to aerosol size and number effects is probably larger than typical for pure homogeneous freezing $\left(n_{i}\right.$ decreases by a factor of 3.5 at the minimum) because we use unusually high concentrations of large particles (see Appendix).

In case MIX $n$ (black curve), we obtain the same result for $n<10^{-3} \mathrm{~cm}^{-3}$ as in the pure HOM case. Although the IN contained in the large mode freeze earlier than the bulk of liquid droplets from the small mode, the number of IN is too small to affect $n_{i}$. Increasing $n$ leads to a decrease in $n_{i}$ that is much more pronounced than in case HOM; a sharp minimum develops at $n=0.025 \mathrm{~cm}^{-3}$, where $n_{i}=n$. This happens because the IN gradually prevent the liquid droplets from freezing, and completely shut off homogeneous freezing exactly at the minimum. While the minimum in case HOM results from a change in the aerosol size distribution only, the cusp-like minimum in case MIX is caused by competitive freezing of externally mixed IN. When $n$ increases further, only IN particles freeze and $n_{i}$ rises strictly in proportion to $n$, because $n_{i}$ is only limited by the availability of IN in this regime up to $n=0.6 \mathrm{~cm}^{-3}$. Later, $n_{i}$ saturates near $n=6 \mathrm{~cm}^{-3}$. The value of $n_{i}$ for $n>10 \mathrm{~cm}^{-3}$ in case MIX $n$ slightly exceeds that in case HOM because of the difference in freezing thresholds (Kärcher and Lohmann, 2003, their Sect. 2.1.3).

The suppression of ice crystals in cirrus due to the addition of IN (negative Twomey effect) has been described previously (Kärcher and Lohmann, 2003, their Sect. 4.2), and leads to a strong decrease of $n_{i}$ by a factor of 28 at the minimum (Fig. 4). The minimum in case MIX $n$ is the minimum concentration of IN above which homogeneous freezing does not take place anymore. However, homogeneous freezing did take place in the NH campaign, as we have shown in Sect. 3.3.2. Moreover, the mean observed $n_{i}$ in the NH cam-

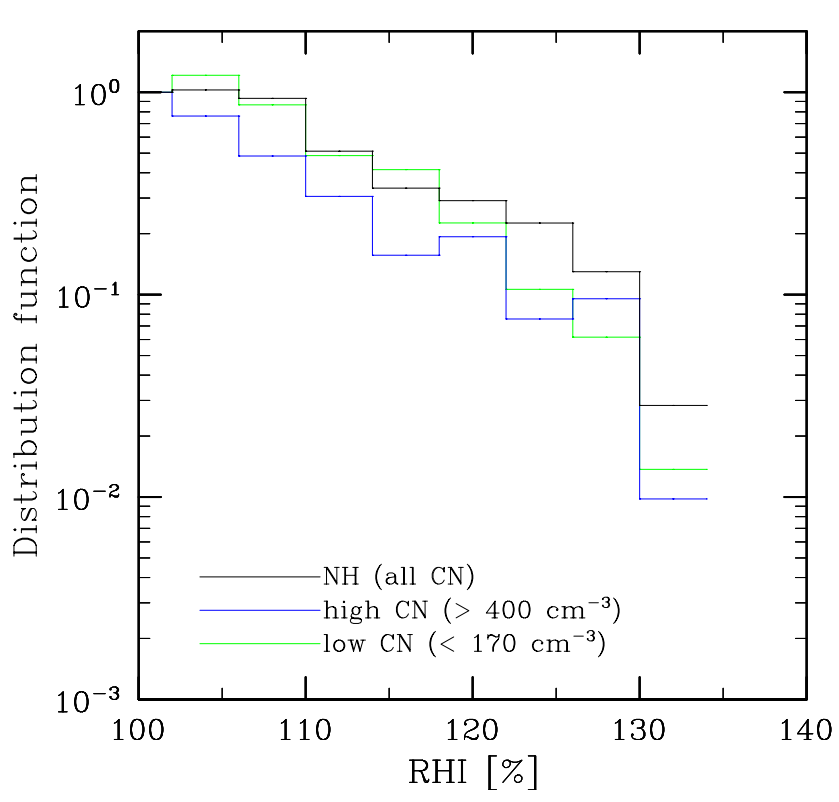

Fig. 5. Distributions of RHI above ice saturation outside of cirrus clouds measured during INCA in Prestwick (NH, black curve, repeated from the left panel of Fig. 2). The green (blue) curve comprise a subset of these data, representing relatively clean (polluted) airmasses with low (high) concentrations of condensation nuclei (CN) larger than $14 \mathrm{~nm}$ in diameter. The $\mathrm{CN}$ concentrations in the clean $\mathrm{NH}$ case are similar to the typical $\mathrm{CN}$ concentrations observed in the $\mathrm{SH}$.

paign was almost identical to that observed in the $\mathrm{SH}$ and consistent with what is expected from homogeneous freezing (Kärcher and Ström, 2003). Together this implies that the actual IN concentrations must have been lower than the above deduced upper limit. Figure 4 suggests that $n_{i}$ is only weakly affected by the presence of IN that freeze at $130 \%$ at IN number densities $<0.01 \mathrm{~cm}^{-3}$.

The above simulations have been carried out with constant cooling rates. The rapid temperature fluctuations often present in cirrus conditions likely introduce scatter in the predicted values of $n_{i}$, rendering a precise determination of an upper limit value consistent with the INCA NH data more difficult. At any rate, the current results shown in Fig. 4 should provide a reasonable estimate. In fact, our deduced IN concentrations $<0.01 \mathrm{~cm}^{-3}$ likely present during the $\mathrm{NH}$ campaign are in very good agreement with existing in situ measurements of IN in the continental upper troposphere at northern midlatitudes (Rogers et al., 1998): highly variable IN concentrations observed with a continuous flow diffusion chamber ranged from $10^{-4}-0.5 \mathrm{~cm}^{-3}$, whereby $60 \%$ of the data fell between $2 \times 10^{-3} \mathrm{~cm}^{-3}$ and $0.02 \mathrm{~cm}^{-3}$.

\section{Speculating about the chemical composition of IN}

Aerosol sources were variable during the $\mathrm{NH}$ campaign; thus one may argue that the freezing threshold of $\sim 130 \%$ 
in the NH is caused by a combination of IN that started freezing very early (near 100\%) and very late (near the homogeneous limit), i.e., not all aerosol particles initiated freezing around $130 \%$ are assumed here. With the help of Fig. 5 we demonstrate that the apparent threshold of $130 \%$ was unlikely to be caused by particles with very different freezing properties.

In Fig. 5, the black distribution is the same as plotted in Fig. 2 (left panel) for the NH measurements. Additionally, we show distributions computed with a subset of the data, representing measurements in clean (green curve) and relatively polluted air masses (blue curve). The distinction between clean and polluted cases has been made on the basis of measured concentrations of condensation nuclei $(\mathrm{CN})$ larger than $14 \mathrm{~nm}$ in diameter. Approximately $30 \%$ of the $\mathrm{NH}$ data are associated with low $\left(<170 \mathrm{~cm}^{-3}\right)$ and high $\left(>400 \mathrm{~cm}^{-3}\right)$ ambient $\mathrm{CN}$ concentrations. We note in passing that the clean $\mathrm{NH}$ cases are similar to the average SH data in terms of $\mathrm{CN}$ concentrations (Minikin et al., 2003).

The three distributions shown in Fig. 5 are almost identical within the range of experimental uncertainty, although they represent conditions with markedly different $\mathrm{CN}$ number densities and, presumably, different IN source regions. The total number of $\mathrm{CN}$ likely contains all of the freezing particles, but may overemphasize the relative contribution of particles that did not freeze (typically more than $90 \%$ of the total). Therefore, we have also analyzed other data subsets, namely accumulation mode particles with diameters above $100 \mathrm{~nm}$ and nonvolatile particles (those remaining after being heated to $250^{\circ} \mathrm{C}$ ) with diameters above $10 \mathrm{~nm}$, and have classified them into clean and polluted cases. The respective number densities were $<10 \mathrm{~cm}^{-3}$ (accumulation mode) and $<10 \mathrm{~cm}^{-3}$ (nonvolatile) in the clean cases and $>20 \mathrm{~cm}^{-3}$ (accumulation mode) and $>40 \mathrm{~cm}^{-3}$ (nonvolatile) in the polluted cases. The results we have obtained are almost identical to those presented in Fig. 5 and are therefore not shown.

We can only speculate about the chemical composition of the IN involved in cirrus formation during INCA. The measurements of light absorbing particles are not yet fully analyzed. From the point of view of global source regions, mineral dust could have been involved in ice formation during the SH campaign, besides liquid particles. The freezing threshold of dust particles immersed in ammoniated sulfate aerosols of $144 \%$ (at $220 \mathrm{~K}$ ) determined in the laboratory (Zuberi et al., 2002) is not inconsistent with our findings, as this value is only $\sim 7 \%$ below the pure homogeneous limit (Kärcher and Lohmann, 2003). Another candidate for nonvolatile material could be sea salt aerosols, expected to be in a fully liquid state at the high observed RHIs (Koop et al., 2000b).

The high concentration of nonvolatile particles present in the $\mathrm{NH}$ campaign may point to black carbon (soot) as a source of IN, especially when previous NH in situ measurements are taken into account (Ström and Ohlsson, 1998). On the other hand, in these previous measurements absorption signatures appeared as banded structures suggesting aged aircraft plumes as the source of soot, while the current analysis shows no sensitivity of the freezing thresholds on the IN source region. Further, the freezing threshold of black carbon particles of $138 \%$ observed in the laboratory (DeMott et al., 1999) is $\sim 8 \%$ above our deduced $\mathrm{NH}$ threshold and was only measured at 5-10 K lower temperatures (Kärcher and Lohmann, 2003). This observation does not rule out soot acting as IN during INCA, as soot particles from different sources may exhibit different freezing properties.

Taken together, these findings have two implications. (1) The deduced NH freezing threshold was likely caused by particles with similar freezing properties, almost independent of their source region, suggesting that even the NH background aerosol exhibits signatures of pollution (expressed here in terms of freezing RHIs below the homogeneous limit). (2) None of the detected differences in aerosol particle concentrations (total $\mathrm{CN}$, accumulation mode, total nonvolatile) between the NH and SH (Minikin et al., 2003) are associated with the differences observed in the distributions of RHI between the two hemispheres. Therefore, differences in chemical or morphological particle properties might have caused the difference in freezing thresholds as presented in Fig. 2. Based on the freezing properties known from laboratory studies and on their source regions, candidates for IN are black carbon $(\mathrm{NH})$ and mineral dust $(\mathrm{SH})$, but definite conclusions cannot be drawn at this point.

Recent analyses of residual particles evaporated from the cirrus ice crystals measured during INCA (Seifert et al., 2003) suggest that the size distribution of particles involved in cirrus nucleation could not possibly be explained by pure homogeneous freezing, supporting the crucial role of sizedependent aerosol chemistry in determining the freezing properties of aerosol particles inferred from Fig. 5.

\subsection{GCM results}

Most global models of the atmosphere that include high-level cirrus clouds apply the saturation adjustment scheme. In this scheme, excess ice supersaturation created during a time step is transferred to cloud ice. Therefore, supersaturation with respect to ice is not allowed to occur in these models. Only recently, the adjustment scheme could be abandoned with the help of a novel parameterization of cirrus cloud formation. This allowed to calculate probability distributions of RHI in ice supersaturated regions along with cirrus clouds formed by homogeneous freezing of supercooled aerosols in a GCM (Lohmann and Kärcher, 2002).

The ECHAM version used here differs from the one previously used for the comparison with relative humidity data from the Measurement of Ozone on Airbus In-service Aircraft (MOZAIC) project. The main change relevant to our study described below is that the number of hygroscopic aerosols that is present in the upper troposphere has been increased by assuming newer observed aerosol size 

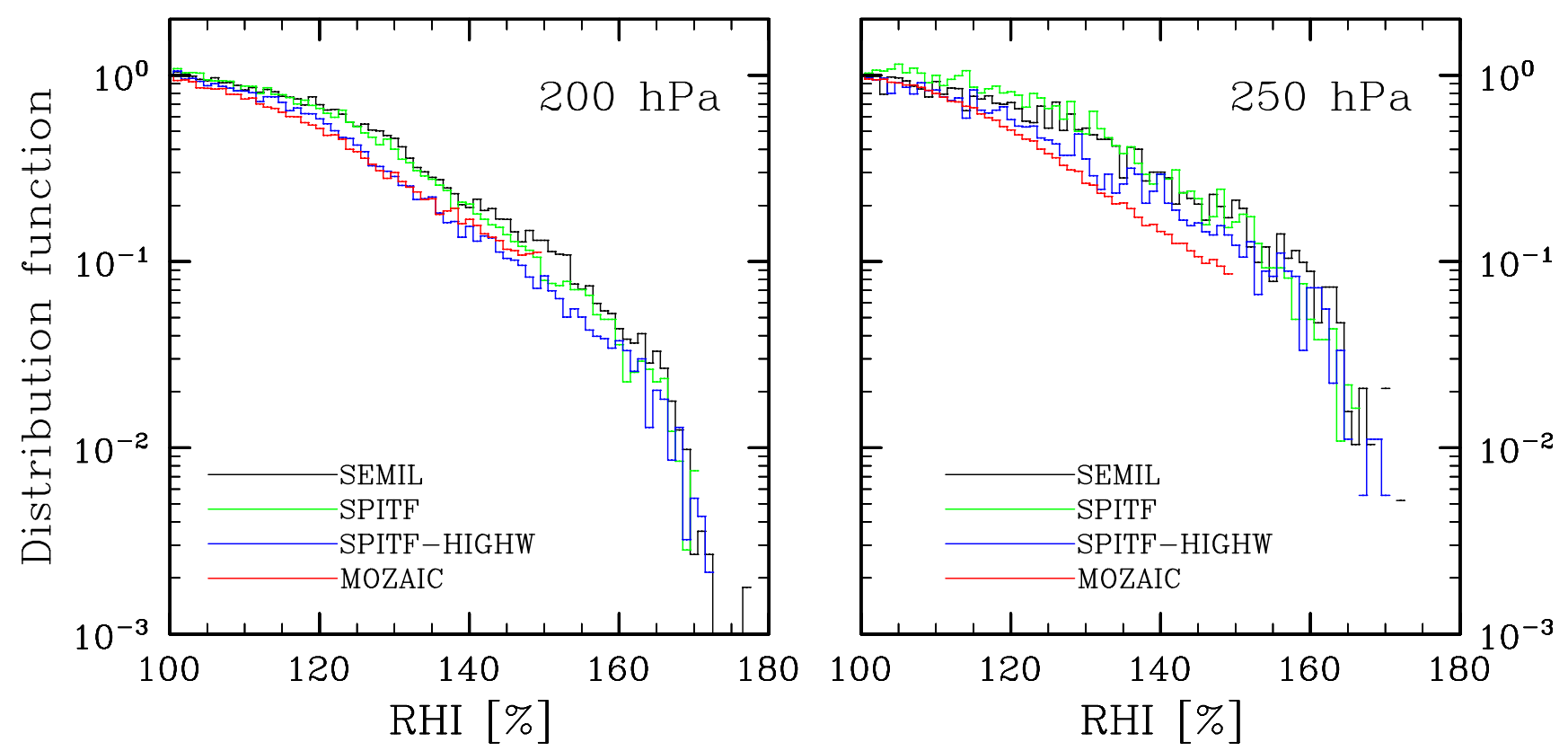

Fig. 6. Distribution of RHI above ice saturation outside of cirrus clouds derived from MOZAIC data and from ECHAM simulations (SEMIL, SPITF, SPITF-HIGHW). Both observed (in the years 1995-1997) and modeled distributions are representative for the North Atlantic region at $200 \mathrm{hPa}$ (left panel) and $250 \mathrm{hPa}$ (right panel). Data from MOZAIC above 150\% have not been analyzed. For details see text.

distributions and increasing the minimum number density of aerosol particles assumed to always be present from 1 to $10 \mathrm{~cm}^{-3}$, in better accordance with observations (Minikin et al., 2003). For a more detailed description, see Lohmann et al. (2003); differently from what has been described there, the advection of cloud droplet and ice crystal number concentrations is now formulated in terms of number of particles per unit mass of air rather than per unit volume of air.

The updraft velocity $w$ needed for ice crystal formation is given as the sum of the grid mean vertical velocity $\bar{w}$ and a turbulent contribution $\Delta w$ obtained from the turbulent kinetic energy (Lohmann and Kärcher, 2002): $w=\bar{w}+c \Delta w$, where $c$ is a tuning parameter. We have shown that this simple approach provides a reasonable estimate for the total number density of cirrus ice crystals $n_{i}$ averaged over a grid box, assuming homogeneous freezing as the dominant pathway to nucleate the cloud particles, but is unable to reproduce the variance of $n_{i}$ in more detail (Kärcher and Ström, 2003).

In the reference simulation SEMIL discussed below, we use a semi-Lagrangian transport scheme (Rasch and Williamson, 1990). No semi-Lagrangian scheme is per se mass conserving due to the interpolation of the Lagrangian departure points onto a regular grid at every time step. Therefore the masses of water vapor, cloud water, and the aerosol mass mixing ratios are conserved by employing a mass fixer. Rasch and Lawrence (1998) developed an improved transport scheme (Spitfire) by introducing a separate mass continuity equation and casting it in flux form. Thus, it now conserves tracer mass and is almost monotonic. It is used in the sim- ulations SPITF and SPITF-HIGHW; the latter case assumes a higher contribution from the turbulent kinetic energy as is used for cloud droplet formation.

Figure 6 depicts distributions of RHI derived from MOZAIC data taken on commercial aircraft mainly over the North Atlantic region (Gierens et al., 1999), along with $f$ (RHI) derived from ECHAM simulations in the same region, assuming homogeneous freezing as the only path to form cirrus clouds. The cloud-clearing criterion used in ECHAM was the same as in the APSC $\left(n_{c}=0\right)$. In ECHAM, we used the semi-Lagrangian advective transport scheme (SEMIL) and the superior transport scheme (SPITF). These two simulations have been carried out with $c=0.7$ to calculate vertical wind speeds. The simulation SPITF-HIGHW assumes a subgrid-scale turbulent contribution about twice as large ( $c=1.33$ ), leading to more frequent cloud formation events and a larger mean number density of ice crystals.

We first focus attention to the left panel, showing data around the $200 \mathrm{hPa}$ level. Compared to case SEMIL, the use of the superior transport scheme SPITF slightly lowers the probability to find $\mathrm{RHI}>120 \%$, keeping the lower values almost unchanged. Increasing the vertical wind speeds, however, reduces the overall probability to find ice supersaturated regions, keeping the slope almost unchanged, compare SPITF with SPITF-HIGHW. While more clouds with higher average crystal concentrations form in case SPITF-HIGHW, the cut-off remains unchanged as we have not altered the underlying homogeneous freezing mechanism.

Generally, the slopes of the SPITF and SPITF-HIGHW cases agree well with the MOZAIC data at $200 \mathrm{hPa}$, but 
the agreement is worse in the $250 \mathrm{hPa}$ level (right panel in Fig. 6). According to the point made at the end of Sect. 3.1, we cannot expect a perfect agreement, inasmuch as the meteorological conditions that prevailed during the airborne measurements are not perfectly similar to the climatological mean calculated by the GCM. Further, the GCM simulations not yet cover all details of real cloud formation processes.

We stress that it is difficult to accurately determine the location of the freezing threshold in the GCM simulations, as subgrid-scale cloud nucleation is parameterized and not calculated explicitly as in the APSC. Because in most cases the total number of ice particles is generated within one time step, and the time step is rather large (30 $\mathrm{min})$, differences between the threshold RHI and the maximum RHI achieved during a freezing event cannot be resolved. Also, RHI is computed every 12 hours from the ECHAM output of $\mathrm{H}_{2} \mathrm{O}$ mixing ratio and $T$ after time integration. This includes the possibility that high RHI values are built up in between, with artificially high values of RHI to be removed in the next time step by freezing and ice particle growth. Therefore, we understand that the thresholds predicted by ECHAM (165$170 \%$, see Fig. 6) may lie close to the actual peak values.

We like to add that the version of Spitfire used in the present study differs from the one used in Lohmann and Kärcher (2002), which unfortunately cannot be repeated. Therefore, the cause of the difference between the simulation SPITF discussed above and the simulation TRANSP in Lohmann and Kärcher (2002) (their Fig. 9) can only be guessed. Very likely, these authors did not use a properly coded Spitfire version as their Spitfire results did not look promising.

\subsection{Other data sets}

In contrast to the modeled distributions shown in Fig. 6, the MOZAIC data set does not exhibit a cut-off, because relative humidities over ice exceeding $150 \%$ have not been analyzed in the measurement analysis owing to the difficulty to separate cloud-free and cloudy cases properly (Klaus Gierens, personal communication, 2002). We have argued in Sect. 3.3.1 that a highly sensitive device is required to isolate the subtle effects of IN on the cloud-free distributions of RHI presented in the present study. Such a cloud-clearing tool was not available.

In any case it is difficult to derive specific conclusions about freezing thresholds and freezing mechanisms from the full set of MOZAIC data, as we have done using the INCA data set. The MOZAIC data are long-term (three year) averages taken in a rather large spatial area. This likely averages out signatures of even significantly different individual freezing thresholds and renders inferences about individual freezing mechanisms almost impossible as very many of them may contribute to the overall distribution.

The distributions of RHI derived from satellite-borne measurements of the Microwave Limb Sounder (MLS) at the
$215 \mathrm{hPa}$ level do not exhibit cut-offs even at high values (180\%) of RHI (Spichtinger et al., 2002), while we argue that a cut-off must be present due to ice formation processes becoming active above a certain freezing threshold. To interpret these data, we note that the instrument has a large field of view (vertical resolution: $\sim 3 \mathrm{~km}$, horiziontal resolution $\sim 100 \times 200 \mathrm{~km}^{2}$ ) and is unable to detect ice crystals smaller than about $100 \mu \mathrm{m}$ in size (typical of particles present in rather young clouds) which could cause retrieved RHI values to be higher than the actual RHI values in the presence of such crystals.

More importantly, the above-mentioned high values have been found only in Antarctic air masses at temperatures much lower than typical midlatitude temperatures. The laboratory data for homogeneous freezing suggest equilibrium freezing thresholds of $165 \%$ for droplet diameters of $0.1 \mu \mathrm{m}$ (Koop et al., 2000a). Hence, non-equilibrium values of up to $180 \%$ may in fact be observable under these cold conditions. Above $180 \%$, the MLS data become very noisy due to a worsening counting statistics.

\section{Conclusions}

We summarize the following main points:

1. The slope of the probability distribution of relative humidity above ice saturation taken close to, but outside of, cirrus clouds is primarily determined by the underlying temperature distribution. Cirrus cloud formation and persistence increases the slope indirectly by reducing supersaturation in regions that would otherwise remain cloud-free.

2. The cut-off of RHI distributions outside of cirrus in ice-supersaturated regions is determined by the onset of freezing of cirrus ice particles, indicating a feasible method to infer freezing threshold relative humidities of cirrus from atmospheric measurements of relative humidity.

3. The comparison between threshold relative humidities outside of cirrus and peak relative humidities inside cirrus is a useful tool to infer details of freezing mechanisms in cirrus clouds.

4. The onset of freezing in the relatively clean midlatitude southern hemisphere during INCA is consistent with homogeneous freezing, although this does not necessarily imply that only pure liquid particles were involved in cloud formation.

5. The onset of freezing in the midlatitude northern hemisphere during INCA occurred at relative humidities over ice significantly lower (by $\sim 24 \%$ ) than required for homogeneous freezing, suggesting that the background aerosol in this region exhibits signatures of pollution in terms of cirrus formation. 
6. The freezing mechanisms at work during INCA have likely involved competition between different types of freezing nuclei, especially in the northern hemisphere. A likely cause for the observed difference in freezing thresholds between the two data sets are differences in chemical or morphological particle properties.

7. The INCA data do not support the notion that ice crystals exclusively form on heterogeneous ice nuclei, stressing the paramount importance of homogeneous freezing in the formation of midlatitude cirrus clouds.

An advantage of our proposed method to infer details of freezing processes from in situ measurements of RHI is that a Lagrangian approach to probe the clouds is not required. Lagrangian measurements are difficult to perform with aircraft. However, only dedicated, short-term field campaigns carried out in a limited area, ideally characterized by uniform sources of aerosol acting as freezing nuclei, allow a meaningful interpretation of RHI distributions, provided (1) enough RHI data points (order 1,000 or more above ice saturation) are available; (2) the data exhibit enough variability in temperature; (3) an accurate cloud-clearing tool is available; and (4) the impact of sedimenting ice crystals in the data set can be minimized or eliminated.

We hope that the results and inferences of this work help improve the representation of cirrus clouds in large-scale models, along with a proper simulation of relative humidity above ice saturation. This is important in the context of atmospheric chemistry, where cirrus clouds may scavenge and vertically redistribute aerosols and reactive trace gases (such as $\mathrm{HNO}_{3}$ or $\mathrm{HO}_{2}$ ) and trigger heterogeneous chemical reactions, as well as in numerical weather prediction models, where the replacement of saturation adjustment schemes by a physically-based parameterization of cirrus formation would help improve important aspects of the radiation balance and the hydrological cycle.

\section{Appendix: Trajectories and microphysical simulations}

We combine the microphysical aerosol/cirrus model APSC (Kärcher, 2003) with three-dimensional trajectories derived from high-resolution ECMWF wind fields with the help of the Lagrangian particle dispersion model FLEXPART (Stohl et al., 1998, 2003).

\section{Trajectory model}

The trajectory calculations were driven with operational ECMWF data, covering the time period September to November 2000. The ECMWF operational forecast model had a spectral truncation of T511 (equivalent to $\sim 40 \mathrm{~km}$ horizontal resolution) and 60 vertical levels. We retrieved the ECMWF data on a global latitude/longitude grid of $1^{\circ}$ resolution. Analyses were used every 6 hours (00:00, 06:00,
12:00, 18:00 UTC) and 3-hour shifted forecasts (at 03:00, 09:00, 15:00, 21:00 UTC) in order to achieve the maximum temporal resolution available from ECMWF.

FLEXPART normally calculates and outputs the concentrations of tracers, based on the advection of a large number of particles that are released at the locations of tracer sources (e.g., pollutant emissions). Here, we have used a new feature of FLEXPART, where particles are initially distributed homogeneously in the atmosphere, according to the distribution of atmospheric mass, and are then followed throughout the simulation ( 3 months in our case, see below). Particle positions were written to output files at regular intervals, such that individual particle trajectories could be followed. While it cannot be claimed that trajectories are accurate over the entire duration of the simulation, we used the trajectory set only in a statistical sense where accuracy is required only for the trajectory segments between the reinitializations of the RHI values. The convection and turbulence schemes of FLEXPART were not used in this study, such that the particle transport was determined solely by the ECMWF winds.

In the original FLEXPART simulations, 160,000 particles, or air parcels, have been evenly distributed over the globe, and their movements have been tracked over a 3-months period, starting 1 September 2000. In the combined FLEXPART/APSC simulations, we only considered particles initially lying within the global midlatitude northern hemisphere $\left(20-80^{\circ} \mathrm{N}\right)$. These particles can leave and reenter this domain and exhibit large vertical displacements. In the microphysical simulations presented below, we ran the APSC model on 10,125 sections of these particle tracks lying within $20-80^{\circ} \mathrm{N}$ and above $400 \mathrm{hPa}$, i.e., we splitted the 3 -months trajectories into shorter segments. This keeps the computational effort for the combined FLEXPART/APSC simulations within manageable bounds and provides sufficient coverage of the computational domain with trajectories over the simulation period. In the final analysis of the RHI data, we only considered data points within the tropopause region (from $2 \mathrm{~km}$ below to $1 \mathrm{~km}$ above the local tropopause).

\section{Microphysical model}

The microphysical simulations were carried out as described by Haag et al. (2003). Freezing and growth processes of aerosol and ice particles were treated using a scheme that exactly conserves the total mass of $\mathrm{H}_{2} \mathrm{O}$ present in the gas and particle phase along a given trajectory, without making equilibrium assumptions. Only water vapor was allowed to interact with the particle phase.

The aerosol size distribution consisted of two lognormal modes, with a total number density of $400 \mathrm{~cm}^{-3}(n)$, a mean number radius (dry particles) of $18 \mathrm{~nm}(420 \mathrm{~nm})$, and a geometric standard deviation of 1.45 (1.3), respectively. While in one case all particles were allowed to freeze homogeneously (referred to as case HOM), we also studied simulations labelled MIX $n$ where the particles in the larger 
mode (with variable total number density $n$ ) acted as efficient ice nuclei (IN). In case HOM, $n$ was fixed at $0.1 \mathrm{~cm}^{-3}$ with the exception of the calculations presented in Sect. 3.3.3 (Fig. 4), where $n$ was varied as in case MIX $n$ for didactic purposes. Finally, the case HET was considered where all the particles from both modes froze heterogeneously, again with $n=0.1 \mathrm{~cm}^{-3}$. The use of $0.1 \mathrm{~cm}^{-3}$ for the concentration of particles in the larger mode can be considered as an extreme case, as such high concentrations of $\mu \mathrm{m}$-sized aerosol particles are probably rare in the tropopause region (Kärcher and Solomon, 1999; Schröder et al., 2002).

The aerosol particles were either composed of aqueous sulfuric acid freezing homogeneously at RHI generally above $145 \%$ according to an activity-based parameterization of nucleation rates (Koop et al., 2000a), or represented some type of ice nuclei (IN) with a prescribed (nominal) heterogeneous freezing threshold of $130 \%$. To calculate heterogeneous nucleation rates, we applied the shifted-activity method described elsewhere (Kärcher and Lohmann, 2003). These rates include the above homogeneous rates as limiting cases. The saturation vapor pressure over ice was taken from Marti and Mauersberger (1993). Ice crystals are assumed to be hexagonal columns with an aspect ratio of 3 for radii $r$ of volume-equivalent spheres above $12.5 \mu \mathrm{m}$, becoming increasingly spherical at smaller sizes (Kärcher, 2003).

We define the nominal heterogeneous threshold of IN such that one particle in equilibrium with ambient $\mathrm{H}_{2} \mathrm{O}$ with $r=0.25 \mu \mathrm{m}$ freezes in $1 \mathrm{~s}$ at a relative humidity over ice of $130 \%$. In the model, depending on the cooling rates at the point of ice formation, particles with very different sizes may contribute to cirrus formation. Therefore we expect a range of freezing thresholds as in the homogeneous case, where the freezing thresholds additionally depend on $T$.

The vertical redistribution of ice crystals (sedimentation) is difficult to treat in a trajectory approach, but is required to correctly simulate cirrus evolution over the entire cloud lifecycle. As a first order approach, we reduce the ice crystal number density in every time step $\Delta t$ by the factor $\exp (-\Delta t / \tau)$ in each size bin. The sedimentation timescale $\tau$ is defined by $\Delta z / v(r)$, with the ice crystal terminal fall speed $v(r)$ and the vertical thickness $\Delta z=1 \mathrm{~km}$. Expressions for $v(r)$ are given by Kärcher (2003). The value of $\Delta z$ has been chosen to bring calculated and observed frequency distributions of the total number densities of ice crystals measured during INCA (Kärcher and Ström, 2003) into good agreement; this issue will be subject of a future publication. As long as we do not extract information about cirrus properties from individual vertical levels, but rather perform statistical analyses of RHI and cloud parameters in the computational domain, we believe that the proposed approach provides a reasonable estimate for the actual loss of ice particles and ice water from the tropopause region.

When RHI was below $50 \%$ along a trajectory, we used a constant time step of $120 \mathrm{~s}$ to advance the calculations. In the range $50 \% \leq \mathrm{RHI} \leq 90 \%, \Delta t$ was reduced to $20 \mathrm{~s}$, and if $\mathrm{RHI}$ exceeded 90\%, $\Delta t$ was further reduced to $4 \mathrm{~s}$. Around the time of freezing, we set $\Delta t=0.25 \mathrm{~s}$. After the ice formation phase, we used a constant time step of $4 \mathrm{~s}$ as long as ice particles were present. The variable time steps ensured that the simulations were fast, as we ran the APSC along ten thousands of trajectories, and accurate, as required to precisely resolve details of water uptake and freezing.

Along each trajectory, we stored the RHI and other microphysical information every $360 \mathrm{~s}$ to mimick uniform sampling of data points, which is required to avoid statistical abberations in the resulting frequency distributions. This requirement is not necessarily met in field measurements, but can easily be accounted for in the calculations. The model output interval of $360 \mathrm{~s}$ was chosen to keep data storage requirements sufficiently small. Data points with nonzero total ice particle number densities were considered as in-cloud data points in the APSC.

Exemplary cirrus calculations including the effects of ice nuclei and mesoscale waves are presented in Kärcher (2003). A comprehensive discussion of our results concerning the physical properties and frequency of cirrus cloud occurrence will be subject of a future publication (Haag and Kärcher, manuscript in preparation).

Acknowledgements. We thank Sabine Eckhardt for processing the ECMWF data used for the trajectory calculations. This research was conducted within the projects "Particles in the Upper Troposphere and Lower Stratosphere and Their Role in the Climate System" (PARTS) and "Interhemispheric Differences in Cirrus Properties From Anthropogenic Emissions" (INCA), funded by the European Commission and contributes to the project "Particles and Cirrus Clouds" (PAZI) supported by the Helmholtz-Gemeinschaft Deutscher Forschungszentren (HGF). Ulrike Lohmann is grateful for support from the project "Modelling of Global Chemistry for Climate" funded by the National Science and Engineering Research Council of Canada and the Canadian Foundation for Climate and Atmospheric Science.

\section{References}

Borrmann, S., Solomon, S., Dye, J. E., and Luo, B.: The potential of cirrus clouds for heterogeneous chlorine activation, Geophys. Res. Lett., 23, 2133-2136, 1996.

Braham, R. R.: Cirrus cloud seeding as a trigger for storm development, J. Atmos. Sci., 24, 311-312, 1967.

Danielsen, E. F.: A dehydration mechansim for the stratosphere, Geophys. Res. Lett., 9, 605-608, 1982.

DeMott, P. J., Rogers, D. C., and Kreidenweis, S. M.: The susceptibility of ice formation in upper tropospheric clouds to insoluble aerosol components, J. Geophys. Res., 102, 19575-19584, 1997.

DeMott, P.J., Chen, Y., Kreidenweis, S. M., Rogers, D. C., and Sherman, D. E.: Ice formation by black carbon particles, Geophys. Res. Lett., 26, 2429-2432, 1999.

Dessler, A. E., Minschwaner, K., Weinstock, E. M., Hintsa, E. J., Anderson, J. G., and Russell, III, J. M.: The effects of tropical 
cirrus clouds on the abundance of lower stratospheric ozone, J. Atmos. Chem., 23, 209-220, 1996.

Gettelman, A., Randel, W. J., Wu, F., and Massie, S. T.: Transport of water vapor in the tropical tropopause layer, Geophys. Res. Lett., 29, 10.1029/2001GL013818, 2002.

Gierens, K. M., Schumann, U., Helten, M., Smit, H., and Marenco, A.: A distribution law for relative humidity in the upper troposphere and lower stratosphere derived from three years of MOZAIC measurements, Ann. Geophys., 17, 1218-1226, 1999.

Haag, W., Kärcher, B., Schaefers, S., Stetzer, O., Möhler, O., Schurath, U., Krämer, M., and Schiller, C.: Numerical simulations of homogeneous freezing processes in the aerosol chamber AIDA, Atmos. Chem. Phys., 3, 195-210, 2003.

Heymsfield, A. J. and Miloshevich, L. M.: Relative humidity and temperature influences on cirrus formation and evolution: $\mathrm{Ob}$ servations from wave clouds and FIRE II, J. Atmos. Sci., 52, 4302-4326, 1995.

Heymsfield, A. J., Miloshevich, L. M., Twohy, C., Sachse, G., and Oltmans, S.: Upper-tropospheric relative humidity observations and implications for cirrus ice nucleation, Geophys. Res. Lett., 25, 1343-1346, 1998.

Jensen, E. J., Toon, O. B., Pfister, L., and Selkirk, H. B.: Dehydration of the upper troposphere and lower stratosphere by subvisible cirrus clouds near the tropical tropopause, Geophys. Res. Lett., 23, 825-828, 1996.

Jensen, E. J., Toon, O. B., Vay, S. A., Ovarlez, J., May, R., Bui, P., Twohy, C. H., Gandrud, B., Pueschel, R. F., and Schumann, U.: Prevalence of ice-supersaturated regions in the upper troposphere: Implications for optically thin ice cloud formation, J. Geophys. Res., 106, 17 253-17 266, 2001.

Kärcher, B.: Simulating gas-aerosol-cirrus interactions: Processoriented microphysical model and applications, Atmos. Chem. Phys., 3, 1645-1664, 2003.

Kärcher, B. and Solomon, S.: On the composition and optical extinction of particles in the tropopause region, J. Geophys. Res., 104, $27441-27459,1999$.

Kärcher, B. and Lohmann, U.: A parameterization of cirrus cloud formation: Homogeneous freezing including effects of aerosol size, J. Geophys. Res., 107, 4698, doi:10.1029/2001JD001429, 2002.

Kärcher, B. and Lohmann, U.: A parameterization of cirrus cloud formation: Heterogeneous freezing, J. Geophys. Res., 108, 4402, doi:10.1029/2002JD003220, 2003.

Kärcher, B. and Ström, J.: The roles of dynamical variability and aerosols in cirrus cloud formation, Atmos. Chem. Phys., 3, 823 838, 2003.

Kärcher, B. and Haag, W.: Factors controlling upper tropospheric relative humidity, Ann. Geophys., 21, in press, 2003.

Koop, T., Luo, B. P., Tsias, A., and Peter, Th.: Water activity as the determinant for homogeneous ice nucleation in aqueous solutions, Nature, 406, 611-614, 2000a

Koop, T., Kapilashrami, A., Molina, L. T., and Molina, M. J.: Phase transitions of sea salt/water mixtures at low temperatures: Implications for ozone chemistry in the polar marine boundary layer, J. Geophys. Res., 105, 26 393-26 402, 2000b.

Lohmann, U. and Kärcher, B.: First interactive simulations of cirrus clouds formed by homogeneous freezing in the ECHAM GCM, J. Geophys. Res., 107, 4105, doi:10.1029/2001JD000767, 2002.

Lohmann, U., Kärcher, B., and Timmreck, C.: Impact of the
Mt. Pinatubo eruption on cirrus clouds formed by homogeneous freezing in the ECHAM GCM, J. Geophys. Res., 108, 4568, doi:10.1029/2002JD003185, 2003.

Marti, J. and Mauersberger, K.: A survey and new measurements of ice vapor pressure at temperatures between 170 and $250 \mathrm{~K}$, Geophys. Res. Lett., 20, 363-366, 1993.

Minikin, A., Petzold, A., Ström, J., Krejci, R., Seifert, M., van Velthoven, P., Schlager, H., and Schumann, U.: Aircraft observations of the upper tropospheric fine particle aerosol in the northern and southern hemispheres at midlatitudes, Geophys. Res. Lett., 30, 1503, doi:10.1029/2002GL016458, 2003.

Ovarlez, J. and van Velthoven, P.: Comparison of water vapor measurements with data retrieved from ECMWF analyses during the POLINAT experiment, J. Appl. Meteorol., 36, 1329-1335, 1997.

Ovarlez, J., Gayet, J.-F., Gierens, K., Ström, J., Ovarlez, H., Auriol, F., Busen, R., and Schumann, U.: Water vapour measurements inside cirrus clouds in Northern and Southern hemispheres during INCA, Geophys. Res. Lett., 29, 1813, doi:10.1029/2001GL014440, 2002.

Pfister, L., Selkirk, H. B., Jensen, E. J., Schoeberl, M. R., Toon, O B., Browell, E. V., Grant, W. B., Gary, B., Mahoney, M. J., Bui, T. V., and Hintsa, E.: Aircraft observations of thin cirrus clouds near the tropical tropopause, J. Geophys. Res., 106, 9765-9786, 2001.

Rasch, P. J. and Williamson, D. L.: Computational aspects of moisture transport in global models of the atmosphere, Q. J. R. Meteorol. Soc., 116, 1071-1090, 1990.

Rasch, P. J. and Lawrence, M.: Recent development in transport methods at NCAR, in MPI Workshop on Conservative Transport Schemes, Machenhauer, B. (Ed), Hamburg, Germany, MaxPlanck-Institute for Meteorology, Report No. 265, pp. 65-75, 1998.

Rogers, D. C., DeMott, P. J., Kreidenweis, S. M., and Chen, Y.: Measurements of ice nucleating aerosols during SUCCESS, Geophys. Res. Lett., 25, 1383-1386, 1998.

Schröder, F., Kärcher, B., Fiebig, M., and Petzold, A.: Aerosol states in the free troposphere at northern midlatitudes, J. Geophys. Res., 107, 8126, doi:10.1029/2000JD000194, 2002.

Seifert, M., Ström, J., Krejci, R., Minikin, A., Petzold, A., Gayet, J.F., Schumann, U., and Ovarlez, J.: In situ observations of aerosol particles remaining from evaporated cirrus crystals: Comparing clean and polluted air masses, Atmos. Chem. Phys., 3, 10371049, 2003.

Spang, R., Eidmann, G., Riese, M., Offermann, D., Preusse, P., Pfister, L., and Wang, P.-H.: CRISTA observations of cirrus clouds around the tropopause, J. Geophys. Res., 107, 8174, doi:10.1029/2001JD000698, 2002

Spichtinger, P., Gierens, K., and Read, W.: The statistical distribution law of relative humidity in the global tropopause region, Meteorol. Z., 11, 83-88, 2002.

Stohl, A., Hittenberger, M., and Wotawa, G.: Validation of the Lagrangian particle dispersion model FLEXPART against largescale tracer experiment data, Atmos. Environ., 32, 4245-4264, 1998.

Stohl, A., Forster, C., Eckhardt, S., Spichtinger, N., Huntrieser, H., Heland, J., Schlager, H., Wilhelm, S., Arnold, F., and Cooper, O.: A backward modeling study of intercontinental pollution transport using aircraft measurements, J. Geophys. Res., 108, 4370, doi:10.1029/2002JD002862, 2003 
Ström, J., and Ohlsson, S.: In situ measurements of enhanced crystal number densities in cirrus clouds caused by aircraft exhaust, J. Geophys. Res., 103, 11355-11361, 1998.

Ström, J., Seifert, M., Kärcher, B., Ovarlez, J., Minikin, A., Gayet, J.-F., Krejci, R., Petzold, A., Auriol, F., Busen, R., Schumann, U., Haag, W., and Hansson, H.-C.: Cirrus cloud occurrence as a function of ambient relative humidity: A comparison of observations obtained during the INCA experiment, Atmos. Chem. Phys., 3, 1807-1816, 2003.
Zuberi, B., Bertram, A. K., Cassa, C. A., Molina, L. T., and Molina, M. J.: Heterogeneous nucleation of ice in $\left(\mathrm{NH}_{4}\right)_{2} \mathrm{SO}_{4}-\mathrm{H}_{2} \mathrm{O}$ particles with mineral dust immersions, Geophys. Res. Lett., 29, 1504, doi:10.1029/2001GL014289, 2002. 